Note

\title{
A note on efficient signaling of bargaining power
}

\author{
Mehmet Bac* \\ Bilkent University, Department of Economics, Bilkent, Ankara, 06533 Turkey \\ (e-mail: bac@bilkent.edu.tr) \\ Received: July 1996/Final version: August 1999
}

\begin{abstract}
Strategic delay and restricted offers are two modes of signaling bargaining power in alternating offers bargaining games. This paper shows that when both modes are available, the best signaling strategy of the "strong" type of the informed player consists of a pure strategic delay followed by an offer on the whole pie. There is no signaling motivation for issue-by-issue bargaining when the issues are perfectly substitutable.
\end{abstract}

Key words: Alternating offers bargaining, incomplete information, sequential equilibrium, delay

\section{Introduction}

The informed player in a standard alternating offers bargaining game has two instruments to signal his bargaining power. The first and the most well-known is what Admati and Perry (1987) have termed strategic delay, a strategy for the strong type that prescribes remaining silent for a sufficiently long period of time and an offer that the weak (impatient or high-valuation) type cannot beneficially imitate. The second signaling instrument is a restricted offer, i.e., an offer restricted to a portion $X$ of the pie which, if accepted, leaves the portion $1-X$ to the next period when the uninformed player takes his turn to make an offer. If $X$ is chosen low enough, the weak type of the informed player prefers making his complete information offer on the whole pie, hence separation occurs after the first round of offers. This result, shown in Bac and Raff (1996), also produces some motivation for bargaining in sequence over

\footnotetext{
* Acknowledgement: I am grateful to an associate editor and especially a referee whose corrections, suggestions and comments greatly improved the paper. Remaining errors are mine.
} 
several issues within a single negotiation because the corresponding bargaining outcome is completed in two steps: an agreement on $X$, followed by an agreement on $1-X$ the next period.

This paper extends the strategies in the standard incomplete information bargaining game to include both strategic delay and restricted offers. Our purpose is to investigate the efficient signaling strategy of the informed player, more precisely, to characterize the combination of strategic delay and restricted offers in a separating bargaining equilibrium. The equilibrium concept is sequential equilibrium, refined with an intuitive condition on beliefs off the equilibrium path.

We consider the first-round separating equilibria of the bargaining game between a seller and an informed buyer, starting with the buyer's offer. In such equilibria the uninformed player learns the type of the informed player after observing the opening offer. Many strategies of the informed player can generate this outcome, i.e., there are many combinations of strategic delay, prices and offer restrictions that the strong buyer type can adopt but the weak buyer type cannot. The analysis identifies a tradeoff between these instruments: The strong buyer type can reduce strategic delay costs by further restricting his offer, but the first mover advantage stipulates that the offer be as unrestricted as possible. We show that strategic delay always dominates in this tradeoff: In the unique first-round separating equilibrium, the strong buyer type delays his offer for a sufficiently long period of time, and then makes an offer on the whole pie. Interpreting the restricted offers outcome as an issue-by-issue bargaining procedure, we conclude that when players are risk neutral and strategic delay is available, there is no signaling motivation for bargaining in sequence over perfectly substitutable issues within a single negotiation.

\section{Efficient signaling in separating bargaining equilibria}

A seller $\mathscr{S}$ owns a perfectly divisible pie. It is common knowledge that the seller's valuation of the pie is zero, while the buyer's valuation $b$ is his private knowledge and can take two values, $H$ (high) or $L$ (low) with $H>L>0$. The high- (low-) valuation buyer is denoted $B_{H}\left(B_{L}\right)$, and the probability that the seller assigns in period $t$ to $b=H$ is denoted $\pi_{t}$.

We study an alternating offer bargaining game that begins in period zero, where it is the informed player $B$ 's turn to make an offer. A player whose turn is to make an offer when $\bar{X}$ of the pie remains chooses a delay $\Delta$ and an offer $(X, P)$ where $P$ is a price (a nonnegative number) and $X \leq \bar{X}$ is an amount of the pie. If the responder accepts this offer, the buyer obtains $X$ and the seller obtains $P$. If $X<1$ (the offer is "restricted") or the responder rejects the offer, a minimal delay occurs and then it is the responder's turn to make an offer. Rubinstein's (1985) bargaining game corresponds to $X=1$ and $\Delta=0$, Admati and Perry's game corresponds to $X=1$ in all offers with $\Delta \geq 0$, whereas in the bargaining game studied by Bac and Raff, $X \in\{0.5,1\}$ and $\Delta=0$ are assumed.

A belief system for $\mathscr{S}$ is a function that specifies a probability to the event $b=H$ given any relevant history of the game. An outcome of the bargaining game involving $N \geq 1$ agreements is denoted $\left(\left(X_{1}, P_{1}, t_{1}\right), \ldots,\left(X_{N}, P_{N}, t_{N}\right)\right)$ where $\sum_{i=1}^{N} X_{i} \leq 1, X_{i} \geq 0$ and $t_{i}$ is the date at which agreement on the portion $X_{i}$ of the pie is reached at the price $P_{i}$. Note that the parties need not 
agree to trade the whole pie at some finite date and that perpetual disagreement is a potential outcome. We consider only pure strategies. The outcome $\left(\left(X_{1}, P_{1}, t_{1}\right), \ldots,\left(X_{N}, P_{N}, t_{N}\right)\right)$ yields the utility $U_{S}=\sum_{i=1}^{N} \delta^{t_{i}} P_{i}$ to the seller, and $U_{b}=\sum_{i=1}^{N} \delta^{t_{i}}\left[b X_{i}-P_{i}\right]$ to the type- $b$ buyer, where $\delta$, with $0<\delta<1$, is the common discount factor.

Our equilibrium concept is sequential equilibrium (see Rubinstein (1985) for a formal definition in the bargaining context), to which we add two restrictions, also imposed by Admati and Perry. The first is a tie-breaking assumption: if a player obtains the same payoff by making fewer offers, then he makes fewer offers. The second restriction concerns beliefs off the equilibrium path: ${ }^{1}$ Fix an equilibrium path and consider a deviation by $B$ after some bargaining history $h$. Call that deviation "bad" for type $b^{\prime}$ if the highest payoff $b^{\prime}$ can so obtain (for any belief the seller might have after observing this deviation) is lower than his continuation equilibrium payoff. The restriction requires that the seller's beliefs put zero probability on type $b^{\prime}$ if there is a type $b$ for whom the deviation in question is not bad. We shall use the term equilibrium for a sequential equilibrium satisfying the above restrictions.

Under complete information, our bargaining game has a unique subgame perfect equilibrium (SPE) where the buyer makes the offer $(1, b \delta /(1+\delta))$. That is, the whole pie is shared immediately and the buyer's corresponding utility is $b /(1+\delta) .{ }^{2}$ Subsequently we assume that the seller's initial belief assigns positive probability to each type of the buyer. In this case, the following lemma, which can be shown using the arguments in Grossman and Perry (1986), provides bounds to the equilibrium utilities of the players determined by the SPE of the complete information version.

Lemma 1. In an equilibrium, after a history in which the amount of the pie remaining is $\bar{X}$,

(i) $\mathscr{S}$ never accepts an offer $(\bar{X}, P)$ with $P<\bar{X} \delta L /(1+\delta)$;

(ii) $B$ never accepts an offer $(\bar{X}, P)$ with $P>\bar{X} H /(1+\delta)$ and always accepts an offer $(\bar{X}, P)$ with $P \leq \bar{X} L /(1+\delta)$.

The following lemma extends Lemma 2.2 in Admati and Perry; the proof follows their arguments.

Lemma 2. Consider a history $h$ that ends in period $t$ with an offer $(X, P)$ by $B$. If $\pi_{t}=0$ (respectively, $\left.\pi_{t}=1\right)$, then in equilibrium $\mathscr{S}$ accepts $(X, P)$ if and only if acceptance yields $\mathscr{S}$ a utility of at least $\delta \bar{X} L /(1+\delta)$ (respectively, $\delta \bar{X} H /(1+\delta))$, where $\bar{X}$ is the amount of the pie remaining when $B$ makes his offer.

Proof. Consider the case $\pi_{t}=0$. By Lemma 1, in equilibrium $\mathscr{S}$ accepts $(X, P)$ only if she obtains at least the utility $\delta \bar{X} L /(1+\delta)$. We need to show that $\mathscr{S}$ accepts $(X, P)$ if doing so yields her a utility of at least $\delta \bar{X} L /(1+\delta)$. Suppose $\mathscr{S}$ rejects such a pair $(X, P)$. Then, there is a continuation equilibrium in which her utility (by the tie-breaking rule) exceeds $\delta \bar{X} L /(1+\delta)$.

1 See Assumption (A2) in Admati and Perry for a formal definition.

2 A proof following the lines of Shaked and Sutton (1984) is available from the author. 
Denote by $U_{S}^{M}$ the supremum of $\mathscr{S}$ 's equilibrium utilities in continuation games following a history in which $\bar{X}$ of the pie remains and $\mathscr{S}$ believes with probability one that $b=L$ and she has just rejected an offer, discounted to the first period in which an offer is subsequently accepted. By assumption, $U_{S}^{M}>\bar{X} L /(1+\delta)$. Choose a continuation equilibrium in which $\mathscr{S}$ obtains the utility $U_{s}^{\sim}$ belonging to the set defined above. Denote the outcome of this equilibrium by $\left(\left(X_{1}^{\sim}, P_{1}^{\sim}, \tau_{1}\right), \ldots,\left(X_{z}^{\sim}, P_{z}^{\sim}, \tau_{z}\right)\right)$.

Suppose that $\mathscr{S}$ makes the first accepted offer $\left(X_{1}^{\sim}, P_{1}^{\sim}\right)$ at date $\tau_{1} \geq t+1$ (the case where $B_{L}$ makes the first accepted offer is similar). The corresponding utilities of $\mathscr{S}$ and $B$, discounted to date $\tau_{1}$, can be written as $U_{\mathscr{\mathscr { S }}}=$ $\sum_{i=1}^{Z} \delta^{\tau_{i}-\tau_{1}} P_{i}^{\sim}$ and $U_{B}^{\sim}=\sum_{i=1}^{Z} \delta^{\tau_{i}-\tau_{1}}\left[b X_{i}^{\sim}-P_{i}^{\sim}\right]=\sum_{i=1}^{Z} \delta^{\tau_{i}-\tau_{1}} b X_{i}^{\sim}-U \tilde{\mathscr{S}}$.

We claim that in period $\tau_{1}$ there is a deviation by $B_{L}$ in which instead of accepting $\mathscr{S}^{\text {'s }}$ offer $\left(X_{1}^{\sim}, P_{1}^{\sim}\right)$ he rejects it, waits until time $\tau_{j}$ and makes a counteroffer $\left(\bar{X}, \bar{U}_{\mathscr{S}}\right)$ for which

$$
\begin{aligned}
& \bar{U}_{\mathscr{S}}>\delta U_{S}^{M}, \\
& L\left(\sum_{i=1}^{Z} \delta^{\tau_{i}-\tau_{1}} X_{i}^{\sim}\right)-U_{\mathscr{S}}^{\sim} \leq \delta^{\tau_{j}-\tau_{1}}\left(L \bar{X}-\bar{U}_{\mathscr{S}}\right), \\
& H\left(\sum_{i=1}^{Z} \delta^{\tau_{i}-\tau_{1}} X_{i}^{\sim}\right)-U_{\mathscr{S}} \geq \delta^{\tau_{j}-\tau_{1}}\left(H \bar{X}-\bar{U}_{\mathscr{S}}\right) .
\end{aligned}
$$

The first inequality implies that $\mathscr{S}$ should accept $B_{L}$ 's deviant offer $\left(\bar{X}, \bar{U}_{\mathscr{S}}\right)$, the second inequality states that $B_{L}$ benefits from the deviation if $\mathscr{S}$ accepts the deviant offer, the third inequality states that $B_{L}$ 's deviation is "bad" for $B_{H}$, and together they imply $\pi_{\tau_{j}}=0$, by the refinement on beliefs. The third inequality above can be written as

$$
\delta^{\tau_{j}-\tau_{1}} \bar{U}_{\mathscr{S}}+H\left[\sum_{i=1}^{Z} \delta^{\tau_{i}-\tau_{1}} X_{i}^{\sim}-\delta^{\tau_{j}-\tau_{1}} \bar{X}\right] \geq U_{\mathscr{S}}^{\sim} .
$$

A necessary condition for (1) to hold is $\sum_{i=1}^{Z} \delta^{\tau_{i}-\tau_{1}} X_{i}^{\sim}>\delta^{\tau_{j}-\tau_{1}} \bar{X}$, which is satisfied for large values of $\tau_{j}$. Since $\bar{U}_{\mathscr{S}}<H \bar{X}$, the LHS of (1) is increasing in $\tau_{j}$ and achieves a limiting value of $H\left[\sum_{i=1}^{Z} \delta^{\tau_{i}-\tau_{1}} X_{i}^{\sim}\right]$. On the other hand, we have $H\left[\sum_{i=1}^{Z} \delta^{\tau_{i}-\tau_{1}} X_{i}^{\sim}\right]-U_{\mathscr{S}} \geq \delta H \bar{X} /(1+\delta)$, for otherwise $B_{H}$ would beneficially deviate. Therefore there exist $U_{\mathscr{S}} \in\left(\bar{X} L /(1+\delta), U_{S}^{M}\right), \bar{U}_{\mathscr{S}}$ such that $\bar{U}_{\mathscr{S}}>\delta U_{S}^{M}$ and a value of $\tau_{j}$ such that (1) holds. The second inequality, which states that $B_{L}$ benefits from the deviation, can be written as

$$
U_{\mathscr{S}}^{\sim} \geq \delta^{\tau_{j}-\tau_{1}} \bar{U}_{\mathscr{S}}+L\left[\sum_{i=1}^{Z} \delta^{\tau_{i}-\tau_{1}} X_{i}^{\sim}-\delta^{\tau_{j}-\tau_{1}} \bar{X}\right]
$$

Note that the RHS of (2) is increasing in $\tau_{j}$, and therefore the relevant point to evaluate (2) is the value of $\tau_{j}$ satisfying (1) with equality when $U_{\mathscr{\varphi}}=$ $U_{S}^{M}$. Also, we can choose $\bar{U}_{\mathscr{S}}$ close enough (almost equal) to $\delta U_{S}^{M}$. Doing this 
evaluation yields the condition $1-L / H \geq \delta^{\tau_{j}-\tau_{1}+1}(1-L / H)$, which is satisfied because $\delta<1$. Thus, all three conditions can simultaneously be satisfied. This contradicts the definition of $U_{S}^{M}$.

The proof for the case $\pi_{t}=1$ uses similar arguments but does not rely on the refinement for beliefs off the equilibrium path. Q.E.D.

Lemma 2 has the following important implication: If $B$ makes his opening offer $(X, P)$ at date $T$ when $\pi_{T}=0$ (respectively, $\pi_{T}=1$ ), in equilibrium $\mathscr{S}$ accepts the offer if and only if acceptance yields her at least the discounted utility $\delta L /(1+\delta)$ (respectively, $\delta H /(1+\delta))$ as viewed from date $T .^{3}$

In what follows, we concentrate on the set of first-round separating equilibria (FRSE) and study the strong buyer's equilibrium signaling strategy.

Definition: $A$ first-round separating equilibrium is an equilibrium such that after the buyer's opening offer the seller either believes that $b=L$ with probability one or believes that $b=H$ with probability one.

We can now state and prove our main result.

Proposition: The game has a unique FRSE. In this equilibrium, $B_{L}$ 's opening offer is $(1, \delta L /(1+\delta))$, which he makes after a delay, and $B_{H}$ 's opening offer is $(1, \delta H /(1+\delta))$, which he makes immediately. S accepts both offers.

Proof. Let "offer $(X, P)$ at date $T_{H}$ " be the first-round action of $B_{H}$ in a FRSE. Thus, when $\mathscr{S}$ receives the offer $(X, P)$, she concludes $\pi_{T_{H}}=1$. By Lemma 2 , in any continuation equilibrium after such a history $\mathscr{S}$ can guarantee herself the discounted utility $\delta H /(1+\delta)$. Therefore, after the history " $(X, P)$ is offered at date $T_{H}$ ", if $\pi_{T_{H}}=1$, the maximum discounted utility that $B_{H}$ can obtain in any continuation equilibrium is $H /(1+\delta)$. Clearly, in equilibrium $B_{H}$ must choose $T_{H}=0$ and offer $(1, \delta H /(1+\delta))$ without delay, which is an offer that $\mathscr{S}$ always accepts. This is the unique opening offer of $B_{H}$ in any FRSE. ${ }^{4}$

We claim that in any FRSE $B_{L}$ makes an accepted opening offer. Suppose, to show a contradiction, that there is a FRE $\sigma$ in which $B_{L}$ makes a rejected opening offer at some date $T_{L}$, and $\pi_{T_{L}}=0$. Let there be $N \geq 1$ agreements $\left(X_{i}, P_{i}\right)_{i=1}^{N}$ dated $T_{1}, T_{2}, \ldots, T_{N}$ where $T_{1}>T_{L}$ along the path of $\sigma$ if $B=B_{L}$. Denote by $U_{\mathscr{S}}^{R}(\sigma)=\sum_{i=1}^{N} \delta^{T_{i}-T_{1}} P_{i}$ the corresponding utility of $\mathscr{S}$, discounted to date $T_{L}$ when she rejects $B_{L}$ 's opening offer. Since $\pi_{T_{L}}=0$, by lemma $2 U_{\mathscr{S}}^{R}(\sigma) \geq \delta L /(1+\delta)$. Since $\sigma$ is a FRSE, $B_{H}$ prefers not to imitate $B_{L}$ 's equilibrium strategy:

$$
\frac{H}{1+\delta} \geq \delta^{T_{1}} H A-\delta^{T_{L}} U_{\mathscr{S}}^{R}(\sigma)
$$

\footnotetext{
${ }^{3} \mathrm{Or}$, if $\pi_{T}=0$ (respectively, $\pi_{T}=1$ ) after the buyer's opening offer at date $T, \mathscr{S}$ rejects the opening offer if and only if acceptance yields her less than $\delta L /(1+\delta)$ (respectively, $\delta H /(1+\delta)$ ) as viewed from date $\mathrm{T}$. This means that at date $\mathrm{T}$, after B's opening offer, in equilibrium $\mathscr{S}$ can guarantee herself the discounted utility $\delta L /(1+\delta)$ if $\pi_{T}=0$ (respectively, $\delta H /(1+\delta)$ if $\left.\pi_{T}=1\right)$. ${ }_{4}^{4}$ Because of discounting, if $B=B_{H}$ it is impossible to construct a FRSE involving two or more agreements such that $B_{H}$ obtains $H /(1+\delta)$ while $\mathscr{S}$ obtains at least $\delta H /(1+\delta)$ as viewed from the date of $B_{H}$ 's opening offer.
} 
where $A=\sum_{i=1}^{N} \delta^{T_{i}-T_{1}} X_{i}$. Consider the following deviation of $B_{L}$ at date $t=$ 0 : Wait until date $T^{D}$ and make the opening offer $(1, P)$ such that

$$
\begin{aligned}
& \frac{H}{1+\delta} \geq \delta^{T_{1}} H A-\delta^{T_{L}} U_{\mathscr{S}}^{R}(\sigma)=\sigma^{T^{D}}(H-P), \\
& \delta^{T_{1}} L A-\delta^{T_{L}} U_{\mathscr{S}}^{R}(\sigma) \leq \delta^{T^{D}}(L-P),
\end{aligned}
$$

and $P \geq \delta L /(1+\delta)$. To see that such a pair $\left(T^{D}, P\right)$ exists, solve for $\delta^{T^{D}}$ from (3) and substitute in (4). Then, (4) can be written as $\delta^{T_{L}} U_{\mathscr{S}}^{R}(\sigma) \geq \delta^{T_{1}} A P$, or

$$
P \leq \frac{U_{\mathscr{P}}^{R}(\sigma)}{\delta^{T_{1}-T_{L}} A} .
$$

Since $U_{\mathscr{I}}^{R}(\sigma) \geq \delta L /(1+\delta), T_{1}>T_{L}$ and $A \leq 1$, there exists $P \geq \delta L /(1+\delta)$ such that (5) holds. Observing the deviant opening offer $(1, P)$ at date $T^{D}$, the seller must believe $\pi_{T^{D}}=0$ and, by lemma 2 , accept $(1, P)$. This contradicts our assumption that $\sigma$ (according to which $B_{L}$ makes a rejected opening offer) is a FRSE.

We now claim that in any FRSE $B_{L}$ 's accepted opening offer involves $X=1$. Suppose, on the contrary, that there is a FRSE $\bar{\sigma}$ which generates the outcome $\left(\left(X_{1}, P_{1}, T_{1}\right), \ldots,\left(X_{N}, P_{N}, T_{N}\right)\right)$ if $B=B_{L}$, where $X_{1}<1$ and $\sum_{i=1}^{N} X_{i} \leq 1$. Let $U_{b}(\bar{\sigma})$ be $B$ 's utility discounted to date $t=0$, and let $U_{\mathscr{S}}^{F}(\bar{\sigma})=\sum_{i=1}^{N} \delta^{T_{t}-T_{1}} P_{i}$ denote $\mathscr{S}$ 's continuation equilibrium utility according to $\bar{\sigma}$, discounted to date $T_{1}$ (first agreement date). We can write $U_{b}(\bar{\sigma})=$ $\delta^{T_{1}}\left[b A-U_{\mathscr{S}}^{F}(\bar{\sigma})\right]$ where $A=\sum_{i=1}^{N} \delta^{T_{i}-T_{1}} X_{i}$. To be a FRSE, $\bar{\sigma}$ must satisfy the following (no imitation) conditions:

$$
\begin{aligned}
& U_{H}(\bar{\sigma}) \equiv \frac{H}{1+\delta} \geq \delta^{T_{1}}\left[H A-U_{\mathscr{S}}^{F}(\bar{\sigma})\right], \\
& U_{L}(\bar{\sigma}) \equiv \delta^{T_{1}}\left[L A-U_{\mathscr{S}}^{F}(\bar{\sigma})\right]>L-\frac{\delta H}{1+\delta},
\end{aligned}
$$

and $U_{\mathscr{S}}^{F}(\bar{\sigma}) \geq \delta L /(1+\delta)$. The first condition states that $B_{H}$ has no incentive to imitate $\bar{\sigma}_{L}$; the second is the corresponding condition for $B_{L}$. In equilibrium, the delay $T_{1}$ must satisfy (6) with equality, which can be solved to yield

$$
\delta^{T_{1}}=\frac{H}{(1+\delta)\left(H A-U_{\mathscr{S}}^{F}(\bar{\sigma})\right)} .
$$

Now define $T\left(A, U_{\mathscr{S}}^{F}(\bar{\sigma})\right)$ as the delay $T_{1}$ that solves (8) as a function of $A$ and the "price" $U_{\mathscr{S}}^{F}(\bar{\sigma}) \geq \delta L /(1+\delta)$. Using (8) in (7), we obtain a reduced form expression for $U_{L}(\bar{\sigma})$ :

$$
U_{L}(\bar{\sigma})=\frac{H\left(L A-U_{\mathscr{S}}^{F}(\bar{\sigma})\right)}{(1+\delta)\left(H A-U_{\mathscr{S}}^{F}(\bar{\sigma})\right)} .
$$

This expression is increasing in $A$ because $U_{L}(\bar{\sigma})<L /(1+\delta)$ (which is implied by the fact that $U_{\mathscr{S}}^{F}(\bar{\sigma}) \geq \delta L /(1+\delta)$ and $\left.A \leq 1\right)$. Therefore $B_{L}$ can 
deviate to the opening offer $\left(1, U_{\mathscr{S}}^{F}(\bar{\sigma})\right)$ at date $T\left(1, U_{\mathscr{S}}^{F}(\bar{\sigma})\right)$. This deviation is "bad" for $B_{H}$, increases $B_{L}$ 's discounted utility above $U_{L}(\bar{\sigma})$, and $\mathscr{S}$ accepts the offer $\left(1, U_{\mathscr{S}}^{F}(\bar{\sigma})\right)$ because $\pi_{T_{L}}=0$ and $U_{\mathscr{S}}^{F}(\bar{\sigma}) \geq \delta L /(1+\delta) .{ }^{5}$ Therefore, in any FRSE, $X_{1}=A=1: B_{L}$ makes his opening offer on the whole pie.

Since $X=1$ in any FRSE, the analysis of the delay-price combination that $B_{L}$ should use in his FRSE opening offer is given by the case $X=1$ studied in Admati and Perry. Lemma 3.1 in their paper shows that when $X=1, B_{L}$ 's equilibrium offer is $(1, \delta L /(1+\delta))$, after delay $\Delta^{*}=T(1, \delta L /(1+\delta))$, given by (8) for $A=1$ and $U_{\mathscr{S}}^{F}(\bar{\sigma})=\delta L /(1+\delta)$.

Let $P_{b}=\delta b /(1+\delta)$. The unique FRSE is given as follows.

$B_{H}$ 's strategy: When $\mathscr{S}$ retains $\bar{X} \leq 1$, offer $\left(\bar{X}, \bar{X} P_{H}\right)$ with no delay; respond to an offer $(X, P)$ as follows:

if $X=\bar{X}$ and $\delta P \leq \bar{X} P_{H}$, accept;

if $0<X<\bar{X}$ and $P+\delta(\bar{X}-X) P_{H} \leq \bar{X} P_{H} / \delta$, accept and offer $\left(\bar{X}-X,(\bar{X}-X) P_{H}\right)$ the next round without delay;

otherwise reject and offer the next round $\left(\bar{X}, \bar{X} P_{H}\right)$ without delay.

$B_{L}$ 's strategy: When $\mathscr{S}$ retains $\bar{X} \leq 1$, offer $\left(\bar{X}, \bar{X} P_{L}\right)$ after the delay $\max \left\{0, T\left(\bar{X}, \bar{X} P_{L}\right)\right\}$. Respond to an offer $(X, P)$ as follows:

if $X=\bar{X}$ and $\delta P \leq \bar{X} P_{L}$, accept;

if $0<X<\bar{X}$ and $L X-P+\delta^{\max \left\{0, T\left(\bar{X}-X,(\bar{X}-X) P_{L}\right)\right\}}\left[(\bar{X}-X) P_{L}\right] \geq$ $\delta^{\max \left\{0, T\left(\bar{X}, \bar{X} P_{L}\right)\right\}} \bar{X} P_{L}$, accept and offer $\left(\bar{X}-X,(\bar{X}-X) P_{L}\right)$ the next round after the delay $\max \left\{0, T\left(\bar{X}-X,(\bar{X}-X) P_{L}\right)\right\}$;

otherwise reject, and offer $\left(\bar{X}, \bar{X} P_{L}\right)$ the next round after the delay $\max \left\{0, T\left(\bar{X}, \bar{X} P_{L}\right)\right\}$.

$\mathscr{S}$ 's strategy: Given the pie stock $\bar{X} \leq 1$ and an offer $(X, P)$ where $X \leq \bar{X}$ made after $T$ units of delay,

(i) if $\pi=1$, accept the offer and counteroffer $\left(\bar{X}-X,(\bar{X}-X) P_{H} / \delta\right)$ if $P+(\bar{X}-X) P_{H} \geq \bar{X} P_{H}$; otherwise reject the offer and counteroffer $\left(\bar{X}, \bar{X} P_{H} / \delta\right)$ with no delay;

(ii) if $\pi=0$, accept the offer and counteroffer $\left(\bar{X}-X,(\bar{X}-X) P_{L} / \delta\right)$ if $P+(\bar{X}-X) P_{L} \geq \bar{X} P_{L}$; otherwise reject the offer and counteroffer $\left(\bar{X}, \bar{X} P_{L} / \delta\right)$ with no delay.

$\mathscr{S}$ 's beliefs: Given the pie stock $\bar{X} \leq 1$, if $B$ makes an offer $\left(X_{1}, P_{1}\right)$ after delay $T_{1}$ such that (6) and (7) hold for $\sum_{i} X_{i} \leq \bar{X}$ and $\sum_{i} \delta^{T_{i}-T_{1}} P_{i} \geq P_{L}$, then $\pi=0$. Otherwise $\pi=1$.

\section{Conclusions}

To recapitulate, in the alternating offers, incomplete information bargaining game with linear buyer preferences $u_{b}=b X$ over portions $X$ of the pie, the only first-round separating equilibrium outcome is $(1, \delta H /(1+\delta), 0)$ if $b=H$ and $(1, \delta L /(1+\delta), T(1, \delta L /(1+\delta)))$ if $b=L$. That is, signaling bargaining power through strategic delay is a more effective strategy than restricted

\footnotetext{
${ }^{5}$ The tradeoff between strategic delay and offer restriction, which is mentioned in the introduction, is apparent in (8) defining $T\left(A, U_{\mathscr{S}}^{F}(\bar{\sigma})\right)$. By choosing $X_{1}=1$, hence $A=1, B_{L}$ can reduce delay costs.
} 
offers. While a restricted offer economizes on delay and the effective price that the strong buyer pays for the whole pie, his relatively low delay cost and the cost of losing the first-mover advantage on the remaining $1-X$ portion of the pie dominate the tradeoff.

An important extension is to drop the assumption of constant marginal utility of the pie, which can be interpreted as allowing buyer types to have heterogeneous preferences over different portions of the pie. Since $u(x+y)<$ $u(x)+u(y)$ for any strictly concave utility function, introducing a decreasing marginal utility for the strong type of the informed player will strengthen the case for restricted offers. We have investigated this question in Bac (2000) and shown that the answer depends on the nature of the "pie": If the pie is a consumption good, under certain conditions the strong buyer's FRSE strategy involves a restricted offer; if the pie is a durable good, then this strategy is unlikely to involve a restricted offer. Another interesting formulation of the bargaining problem would be to consider two pies (or "issues") on which the players may have sub-additive or super-additive preferences. Under complete information, Fershtman (1990) has shown that such differing preferences over individual pies play a role in determining negotiation agendas. It would be interesting to see how multiple signaling modes interact under differing preference structures.

\section{References}

Admati A, Perry M (1987) Strategic delay in bargaining. Review of Economic Studies 54:345-364

Bac M (2000) Signaling bargaining power: Strategic delay versus restricted offers. Economic Theory, forthcoming

Bac M, Raff H (1996) Issue-by-issue negotiations: The role of information and time preference. Games and Economic Behavior 13:125-134

Fershtman C (1990) The importance of the agenda in bargaining. Games and Economic Behavior 2:224-238

Grossman S, Perry M (1986) Sequential bargaining under asymmetric information. Journal of Economic Theory 39:120-154

Rubinstein A (1985) A bargaining model with incomplete information about preferences. Econometrica 50:1151-1172

Shaked A, Sutton J (1984) Involuntary unemployment as a perfect equilibrium in a bargaining model. Econometrica 52:1351-1364 